\title{
Individual and neighborhood socioeconomic status and progressive chronic kidney disease in an elderly population: The Cardiovascular Health Study
}

\author{
Sharon Stein Merkin ${ }^{\mathrm{a}, *, 1}$, Ana V. Diez Roux ${ }^{\mathrm{b}}$, Josef Coresh ${ }^{\mathrm{a}}$, Linda F. Fried ${ }^{\mathrm{c}}$, \\ Sharon A. Jackson ${ }^{\mathrm{d}}$, Neil R. Powe ${ }^{\mathrm{a}}$ \\ a Johns Hopkins Bloomberg School of Public Health, The Welch Center for Prevention, \\ Epidemiology and Clinical Research, Blatimore, MD 21205, USA \\ ${ }^{\mathrm{b}}$ University of Michigan School of Public Health, MI 48128, USA \\ ${ }^{\mathrm{c}}$ VA Pittsburgh Healthcare System, University of Pittsburgh School of Medicine, Pittsburgh, PA 15260, USA \\ ${ }^{\mathrm{d}}$ Wake Forest University School of Medicine, Winston-Salem, NC 27157, USA
}

Available online 11 May 2007

\begin{abstract}
Few studies have focused on the association between socioeconomic status (SES) and progressive chronic kidney disease (pCKD) in an elderly population. We conducted a cohort study of 4735 Cardiovascular Health Study participants, ages 65 and older and living in 4 US communities, to examine the independent risk of pCKD associated with income, education and living in a low SES area.

pCKD was defined as creatinine elevation $\geqslant 0.4 \mathrm{mg} / \mathrm{dL}(35 \mu \mathrm{mol} / \mathrm{L})$ over a $4-7$ year follow-up or CKD hospitalization. Area SES was characterized using measures of income, wealth, education and occupation for 1990 (corresponding to time of enrollment) US Census block groups of residence.

Age and study site-adjusted incidence rates (per 1000 person years) of pCKD by quartiles of area-level SES score, income and education showed decreasing rates with increasing SES. Cox proportional hazards models showed that living in the lowest SES area quartile, as opposed to the highest, was associated with $50 \%$ greater risk of pCKD, after adjusting for age, gender, study site, baseline creatinine, and individual-level SES. This increased risk and trend persisted after adjusting for lifestyle risk factors, diabetes and hypertension. We found no significant independent associations between pCKD and individual-level income or education (after adjusting for all other SES factors). As such, living in a low SES area is associated with greater risk of pCKD in an elderly US population.
\end{abstract}

(C) 2007 Elsevier Ltd. All rights reserved.

Keywords: Socioeconomic; Kidney disease; Neighborhood; Elderly; USA

\footnotetext{
*Corresponding author. Tel.: + 13108258253 .

E-mail address: smerkin@mednet.ucla.edu (S.S. Merkin).

${ }^{1}$ Currently at the UCLA Geffen School of Medicine, Division of Geriatrics, USA.
}

\section{Introduction}

Chronic kidney disease (CKD) is a significant public health burden in the United States that is especially relevant in the elderly population. According to the United States Renal Data System 
(USRDS), the incidence rate of end-stage renal disease (ESRD) was 336 per million in 2002 (US Renal Data System, 2004) and is projected to rise to 612 per million by 2010 (U.S. Department of Health and Human Services, 2000). Incidence of ESRD increases dramatically with age, with those over age 65 exhibiting the highest rates of disease; these rates were reported in 2002 as 1308/million for those ages 65-74 and 1456/million for people 75 year and older (US Renal Data System, 2004). If left untreated, mild forms of kidney impairment can progress to ESRD. Exploring the risk factors related to kidney disease progression in an elderly population is thus essential to reducing morbidity and mortality related to CKD.

Low socioeconomic status (SES) has been found to adversely affect the risk of kidney disease, when examining individual (Krop et al., 1999; Perneger, Whelton \& Klag, 1995; Rostand, Brown, Kirk, Rutsky \& Dustan, 1989) or area SES (Brancati, Whittle, Whelton, Seidler \& Klag, 1992; Byrne, Nedelman, \& Luke, 1994; Klag et al., 1997; New York State Department of Health, 1991; Rostand, 1992; Whittle, Whelton, Seidler, \& Klag, 1991; Young, Mauger, Jiang, Port, \& Wolfe, 1994). In a recent study, we found a strong association between living in a low SES area and progressive chronic kidney disease (pCKD) among white middle-aged men (Stein Merkin, Coresh, Diez Roux, Taylor, \& Powe, 2005). To our knowledge, that study was the first to examine CKD progression (pre-end stage) and area-level SES. Moreover, few studies have examined the associations of SES (individual or area) with kidney disease in an elderly population. To the extent that low socioeconomic position in the elderly is a marker for a history of low SES over the lifecourse, SES in the elderly may reflect cumulative exposure to conditions associated with SES and also associated with the development of CKD, such as hypertension and diabetes. Low socioeconomic position may also be a marker for access to treatment and poor control of hypertension and diabetes.

Several different dimensions of individual-level SES may be relevant to the development of CKD. This study focuses on two common measures of individual-level SES available in epidemiologic studies: income and education. Income is a measure of monetary resources and purchasing power. Income may affect access to optimum health care, medical visits and medication and may also affect a person's ability to adopt and maintain a healthy diet and physical activity. Education directly affects job attainment and income. In addition, education can influence knowledge about disease risk factors (Davis, Ahn, Fortmann \& Farquhar, 1998; Winkelby, Jatulis, Frank \& Fortmann, 1992) and the health care system, as well as the ability to utilize it effectively (Pincus, 1988; Sabates \& Feinstein, 2006).

Area-based measures of SES may also be of special relevance to the elderly for several reasons. Place of residence in old age may be a better marker for SES over the lifecourse than measures like current income, which often decline in old age (Kaplan, Seeman, Cohen, Knudsen, \& Guralnik, 1987). In addition, there may be different mechanisms through which area characteristics may be related to the risk of pCKD aside from individuallevel socioeconomic position. Low socioeconomic neighborhoods have been found to be associated with a lack of physical activity facilities (GordonLarsen, Nelson, Page \& Popkin, 2006; Powell, Slater, Chaloupka \& Harper, 2006) and limited access to healthy and affordable food (Horowitz, Colson Hebert, \& Lancaster, 2004; Moore \& Diez Roux, 2006; Powell, Slater, Mirtcheva, Bao, \& Chaloupka, 2007). In addition, studies have shown that individuals living in low SES areas are less likely to seek adequate and preventive health care (Pappas, Hadden, Kozak, \& Fisher, 1997). Considering the importance of these factors with regard to controlling and avoiding the major risk factors for kidney disease, diabetes and hypertension, these neighborhood conditions may play a role in the progression of CKD. These area characteristics may be of special relevance to the elderly, since older individuals are more likely to spend time in their neighborhoods and to rely on local areas for resources and services (Robert \& Li, 2001), including health care. Area characteristics may also be proxies for harmful environmental exposures including nephrotoxins (Lin, Tan, Hsu, \& Yu, 2001; Steenland, Thun, Ferguson, \& Port, 1990).

The goal of this study was to examine the independent associations of individual and arealevel SES with pCKD in an elderly population.

\section{Methods}

\section{Study design and population}

The study population consists of participants in the Cardiovascular Health Study (CHS), a longitudinal population-based study of cardiovascular 
disease, including coronary heart disease and stroke. Participants were randomly sampled from Medicare eligibility lists in 4 US communities, including Forsyth County, North Carolina; Washington County, Maryland; Sacramento County, California; and Pittsburgh (Allegheny County), Pennsylvania (once participants were sampled, spouses were also invited to participate). A total of 5888 men and women were recruited, ages 65 and older. The initial cohort was recruited between 1989 and $1990(n=5201)$, and an additional African American cohort $(n=687)$ was recruited from the counties in NC, CA and PA in 1992. Although participants were actively followed-up each year, data for this study were obtained from the baseline visit and follow-up visits that included serum creatinine measurement: baseline (1989-1990), 3rd year follow-up (1992-1993), and 7th year follow-up (1996-1997). Since the second cohort was only recruited in 1992, that year was considered baseline and the "7th year" was the only follow-up visit for the second cohort. A detailed description of the methods for the CHS study has been published (Fried et al., 1991; Tell et al., 1993).

\section{Eligibility criteria}

Of the original 5888 participants, 970 were excluded from this study because their addresses were either not successfully geocoded or matched to census areas with unreliable SES data (areas with a population $\leqslant 100,<30$ housing units and where $\geqslant 33 \%$ of the population live in groups quarters). Of the remaining 4918, an additional 33 were excluded because they reported their race as being neither "white" nor "black/African American," and another 9 African American Washington county participants were excluded because the small number made estimates for this group unreliable. An additional 141 participants were excluded because they were missing individual-level education information $(n=16)$, serum creatinine measurement at baseline $(n=71)$, or had serum creatinine levels $\geqslant 2.0 \mathrm{mg} / \mathrm{dL}$ at baseline (to exclude severe kidney impairment at baseline; $n=54$ ). A total of 4735 participants were included in these analyses, representing $80 \%$ of the original cohort.

\section{Incidence of $p C K D$}

Incidence of pCKD was defined as either (1) serum creatinine increase $\geqslant 0.4 \mathrm{mg} / \mathrm{dL}$ at any follow-up visit compared to baseline, or (2) a hospitalization discharge or death coded for conditions related to CKD. An incident event was considered as either of these conditions, whichever occurred first. CKD hospitalizations or deaths included codes for chronic renal disease (International Classification of Diseases, 9th Revision (ICD9) codes 581-583 or 585-588), diabetes with renal manifestations (ICD9 code 250.4), hypertensive renal disease (ICD9 code 403), hypertensive heart and renal disease (ICD9 code 404), or unspecified disorder of kidney and ureter (ICD9 code 593.9). Hospitalizations with codes indicating kidney transplant, renal dialysis or adjustment/ fitting of dialysis catheter (ICD9 codes V42, V45.1, V56, or procedure codes 39.95 , 54.98) were also included.

Serum creatinine data were calibrated to the Cleveland Clinic data indirectly, using NHANES data; we thus subtracted 0.11 and $0.04 \mathrm{mg} / \mathrm{dL}$ from serum creatinine values for the original and second cohort, respectively, to obtain more accurate measures of glomerular filtration rate (GFR, the most commonly accepted clinical measure of kidney function) (Shlipak et al., 2005). GFR is a measure of the kidney's ability to filter the blood, thus removing toxic wastes from the blood. A rise in serum creatinine was defined as an elevation $\geqslant 0.4 \mathrm{mg} / \mathrm{dL}$, based on previous analyses examining kidney function decline (Krop et al., 1999; Muntner, Coresh, Smith, Eckfeldt, \& Klag, 2000; Rostand et al., 1989). One other study examining serum creatinine changes in CHS considered elevations $\geqslant 0.3 \mathrm{mg} / \mathrm{dL}$ (Bleyer, Shemanski, Burke, Hansen, \& Appel, 2000). In an older population, serum creatinine levels may be lower due to declining muscle mass, and thus an even smaller elevation in creatinine may indicate kidney damage. The definition used in this study was, therefore, a conservative one. Hospitalizations and deaths were identified through CHS surveillance throughout the study period with phone calls every 6 months, and included review of hospital and physician records, death certificates, and autopsy and coroner's forms. To ensure completeness of hospitalization records, data were checked against Medicare claims data to identify any hospitalizations that were not reported by the participant.

A pCKD event corresponded to one of the following dates, according to the first occurrence: (1) visit where rise in creatinine was detected (2) date of hospital discharge (3) date of death. Follow-up 
time extended until 6/30/1997, the date marking the end of 7 th year of follow-up (last possible visit for this study). We also conducted sensitivity analyses that included follow-up through 5/31/1999, thus including events that were identified beyond visit dates based on passive surveillance of CKD hospitalizations and deaths, as defined above.

\section{Independent variables}

\section{Individual-level SES}

Individual SES included income and education, as self-reported in the baseline questionnaire. Participants were asked to select their combined family income from a list of 8 categories: $<\$ 5000$, \$5-7999, \$8-11,999, \$12-15,999, \$16-\$24,999, $\$ 25-\$ 34,999, \$ 35-\$ 49,999, \$ 50,000$ or more. For much of the analysis, 4 categories of income were used, approximating quartiles of the income distribution: <\$12,000, \$12-24,999, \$25-34,999, and $\geqslant \$ 35,000$. Education was reported as highest grade or year of school completed, and classified into 5 categories (representing meaningful education credentials/milestones): high school not completed; high school or general equivalency diploma completed; 1-3 years of college; 4 years of college completed; some graduate/professional school. Education was further collapsed into 4 categories for some of the analyses, by combining complete college and graduate education categories.

\section{Area-level SES}

Area-level SES was assigned by geocoding each participant's home address (as reported on the baseline questionnaire) to 1990 US Census block groups. Block groups are contiguous areas defined by the Census Bureau and meant to be homogeneous with respect to living conditions, population characteristics and economic status and include an average of 1000 people (US Bureau of the Census, 1990). Census-based SES measures were assigned to each participant's block group area based on the 1990 Census summary tape file 3 (US Bureau of the Census, 1982).

A summary measure of area-level SES was constructed by summing $z$ scores for 6 censusderived SES indicators, including: median household income; median value of housing units; percent households with interest, dividend or rental income; percentage of residents over age 25 with complete high school education; percentage of residents over age 25 with complete college education; percentage of residents in executive, managerial, or professional specialty occupations. Summary scores ranged from -13.7 to 13.4 in this study sample, with a higher score reflecting a higher SES for a geographic area. The rationale for the combination of these indicators has been described in previous analyses of arealevel SES in the CHS study population (Diez Roux et al., 2001). These six components comprising the SES score, representing income, wealth, education and occupation, reflect a geographic area's physical and social resources. Although these measures are based on aggregate individual information, they represent contextual variables that characterize an area rather than a particular person (Diez-Roux, 1998). Value ranges for the quartiles correspond to: Q1:-13.7 to $-3.1, \mathrm{Q} 2:-3.1$ to $0.3, \mathrm{Q} 3: 0.3$ to 4.0 , Q4: 4.0 to 13.4 .

\section{Other independent variables}

Additional independent variables were considered as potential mediators of the SES-pCKD relationship. We considered smoking, physical activity and obesity, lifestyle risk factors that have been shown to be associated with kidney disease, as well as with low SES (Hallan et al., 2006; Sundquist, Malmstrom \& Johansson, 1999). These behavioral variables were self-reported in the baseline questionnaire or measured at the baseline examination. They were classified as cigarette smoking status (current or former, vs. never), total kcals of physical activity (Taylor et al., 1978), number of blocks walked in past week, and obesity (based on a measure of body-mass-index (BMI; weight $/$ height $^{2}$, $\mathrm{kg} / \mathrm{m}^{2}$ ) $\geqslant 30$ ) (World Health Organization, 2000).

In addition, we considered the two major risk factors for kidney disease, hypertension (systolic blood pressure $\geqslant 140$ or diastolic blood pressure $\geqslant 90$, or a reported history of hypertension and use of antihypertensive medication) and diabetes (fasting glucose $\geqslant 126$, or use of insulin or oral hypoglycemics).

\section{Analysis}

\section{Descriptive analyses}

Descriptive measures of the relevant variables were used to compare the included and excluded study samples. Differences in independent variables were assessed using $\chi^{2}$ tests (for categorical variables), and $t$-tests (for continuous variables). 
Incidence rates of pCKD were calculated based on person years of follow-up. Rates were adjusted for age at baseline and study site based on the overall distribution of age and gender in the entire population, using Poisson regression. Incidence rates were calculated by quartiles of area-level score, and approximated quartiles of individuallevel income and education. $p$-values for trend were calculated by including the SES variables as ordinal variables in the model.

\section{Multivariate analyses}

Cox proportional hazards models were used to calculate hazard ratios of pCKD. All Cox proportional hazards models were fit with robust variance estimation to account for potential clustering by area (block group), unaccounted for by the variables introduced in the model (Lin \& Wei, 1989). Separate basic models were constructed to examine area-level SES, and individual-level income and education categories, in order to examine the effects of each SES indicator on pCKD. The main independent variables for these models included quartiles of area-level score, 4 categories of individual-level income and 4 categories of education (see Methods for values). The highest SES category served as the reference, and adjustments were made for baseline age, race, study site, gender, and baseline serum creatinine. Tests for trend were conducted by including the SES variable in the model as an ordinal variable.

Considering the possibility that area level effects might be stronger among those with low individuallevel SES, interaction terms were considered in the basic models to assess the potential interaction of area and individual SES with respect to risk of pCKD; these interaction terms were tested using the likelihood ratio test to compare models with and without these terms.

Finally, three additional sets of 3 models were fit to examine income, education and area-level SES and their effects on pCKD. These models included incremental adjustments for all the individual-level SES indicators (and in individual-level SES models, for area-level SES), lifestyle risk factors, and then diabetes and hypertension. The independent risk of pCKD associated with area and individual SES was thus observed, and any risk attenuation indicated contributions of the adjusted variables to SES differences.
All reported $p$-values were two-tailed, and significance was ascribed to $p$-values $<0.05$, unless otherwise noted. All analyses were conducted using SAS v.8 (SAS, Release 8.01).

\section{Sensitivity analyses}

Sensitivity analyses considered alternative measures of kidney function to address the limitations associated with using serum creatinine measurements and to examine the separate elements of our combined outcome.

Due to decreased muscle mass at later ages, creatinine clearance in the elderly can underestimate kidney function (Cockcroft \& Gault, 1976). We thus estimated kidney function using a prediction equation for GFR that accounts for age as well as gender and race (Levey et al., 1999; National Kidney Foundation, 2002).

$$
\begin{aligned}
& \text { Estimated GFR }\left(\mathrm{ml} / \mathrm{min} / 1.73 \mathrm{~m}^{2}\right) \\
& \quad=186.3(\text { serum creatinine })^{-1.154}(\text { age })^{-0.203} \\
& \times(0.742 \text { if female })(1.21 \text { if African American }) .
\end{aligned}
$$

Using this equation, we defined CKD as GFR $<60$, based on the National Kidney Foundation $\mathrm{K} / \mathrm{DOQI}$ clinical practice guidelines (National Kidney Foundation, 2002).

We also considered incident pCKD defined exclusively by hospitalizations and deaths. In addition, we considered an alternate method of follow-up ascertainment, where participants were followed for events until the end of the surveillance period (May 31, 1999).

\section{Results}

\section{Characteristics of study population}

Table 1 shows the distribution of selected characteristics by the included and excluded study sample. The excluded group was slightly older, a higher percentage were male and recruited from Washington County. Excluded participants were worse off with regard to all the SES measures considered. In addition, those excluded were also worse off with regard to kidney disease measures, including incidence of pCKD. This was expected, considering that those with advanced kidney disease at baseline were among those excluded. 
Table 1

Distribution of select characteristics by race, The Cardiovascular Health Study

\begin{tabular}{|c|c|c|c|}
\hline Characteristics & $\begin{array}{l}\text { Study population } \\
(n=4735)\end{array}$ & $\begin{array}{l}\text { Excluded population } \\
(n=1153)\end{array}$ & $P$-value \\
\hline Mean baseline age (SD) & $72.7(5.6)$ & $73.3(5.8)$ & 0.002 \\
\hline Gender \% (male) & 41.8 & 44.9 & 0.05 \\
\hline \multicolumn{4}{|l|}{ Race \% } \\
\hline White & 83.7 & 83.3 & \multirow[t]{2}{*}{$<0.0001$} \\
\hline African American & 16.3 & $\begin{array}{l}13.3 \\
3.4 \text { (other race) }\end{array}$ & \\
\hline \multicolumn{4}{|l|}{ Cohort $\%{ }^{\mathrm{a}}$} \\
\hline 1 & 88.2 & 89.1 & \multirow[t]{2}{*}{0.4} \\
\hline 2 & 11.9 & 10.9 & \\
\hline \multicolumn{4}{|l|}{ Study site \% } \\
\hline Forsyth & 27.0 & 23.1 & \multirow[t]{4}{*}{$<0.0001$} \\
\hline Sacramento & 30.3 & 9.4 & \\
\hline Washington & 16.5 & 45.2 & \\
\hline Pittsburgh & 26.2 & 22.4 & \\
\hline \multicolumn{4}{|l|}{ Income $\%$} \\
\hline$<\$ 5,000$ & 4.5 & 6.0 & \multirow[t]{9}{*}{$<0.0001$} \\
\hline$\$ 5,000-\$ 7999$ & 8.8 & 9.7 & \\
\hline$\$ 8,000-\$ 11,999$ & 11.3 & 10.9 & \\
\hline$\$ 12-\$ 15,999$ & 15.2 & 11.5 & \\
\hline$\$ 16-\$ 24,999$ & 18.5 & 17.7 & \\
\hline$\$ 25-\$ 34,999$ & 14.9 & 12.4 & \\
\hline$\$ 35-\$ 49,999$ & 9.5 & 8.5 & \\
\hline$\geqslant \$ 50,000$ & 11.0 & 16.6 & \\
\hline Missing & 6.4 & 6.7 & \\
\hline \multicolumn{4}{|l|}{ Education $\%$} \\
\hline Incomplete high school & 28.7 & 32.3 & \multirow[t]{6}{*}{$<0.0001$} \\
\hline Complete high school/GED/vocational & 37.3 & 31.1 & \\
\hline $1-3$ years college & 14.3 & 12.2 & \\
\hline 4 years college & 10.0 & 11.9 & \\
\hline Graduate school & 9.7 & 11.1 & \\
\hline & & 1.5 (missing) & \\
\hline \multicolumn{4}{|l|}{ Area-level socioeconomic status median } \\
\hline Summary score & 0.32 & $-2.8 \mathrm{~b}$ & $<0.0001$ \\
\hline Median household income & 29,853 & 22,471 & 0.002 \\
\hline Median house value & 85,900 & 76,100 & 0.002 \\
\hline Percent households receiving interest, dividend or rental income & 0.46 & 0.34 & $<0.0001$ \\
\hline Percent people $\geqslant 25$ receiving complete high school education & 0.82 & 0.71 & $<0.0001$ \\
\hline Percent people $\geqslant 25$ receiving $\geqslant$ complete college education & 0.21 & 0.10 & 0.0001 \\
\hline Percent employed in managerial or professional specialty & 0.29 & 0.17 & $<0.0001$ \\
\hline Block groups \# & 889 & 232 & \\
\hline Baseline Serum Creatinine mg/dL: mean (SD) & $0.94(0.27)$ & $1.1(0.72)^{\mathrm{b}}$ & $<0.0001$ \\
\hline Baseline estimated GFR $\mathrm{ml} / \mathrm{min} / / 1.73 \mathrm{~m}^{2}:$ mean $(\mathrm{SD})^{\mathrm{c}}$ & $79.8(23.3)$ & $75.2(25.8)^{\mathrm{b}}$ & $<0.0001$ \\
\hline Mean follow-up years (SD) & $6.4(1.9)$ & $6.1(2.2)^{\mathrm{b}}$ & 0.002 \\
\hline $\begin{array}{l}\text { Age and study site-adjusted incidence rates of } \mathrm{pCKD} / 1000 \text { person } \\
\text { years }(95 \% \mathrm{CI})\end{array}$ & $14.0(12.7-15.4)$ & $21.8(17.7-26.8)^{\mathrm{b}}$ & \\
\hline
\end{tabular}

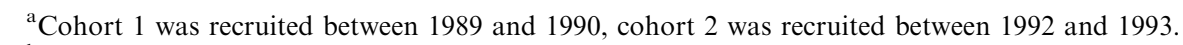

${ }^{\mathrm{b}}$ Sample sizes reduced in the excluded population due to missing values. Sample sizes were $n=1042$ (SES area score), $n=1073$ (baseline creatinine), $n=1036$ (baseline GFR).

${ }^{\mathrm{c}}$ Estimated GFR $\left(\mathrm{ml} / \mathrm{min} / 1.73 \mathrm{~m}^{2}\right)=186.3$ (serum creatinine $)^{-1.154}(\text { age })^{-0.203}(0.742$ if female)(1.21 if African American). 


\section{SES and incidence of $p C K D$}

Decreasing incidence rates of pCKD significantly corresponded to increasing level of area-level SES quartiles (Table 2; 18.1/1000 for the lowest area-level quartile, compared to 10.3 for the highest, $p$-value for trend $=<0.0001)$. The trends were not statistically significant for individual-level SES measures of income or education. However, incidence rates of pCKD at the lowest income and education levels were greater than those at the highest levels (16.1 vs. 12.8 for income, $p$-value for trend $=0.06 ; 17.2$ vs. 12.9 for education, $p$-value for trend $=0.09$ ).

\section{Multivariate analyses}

Table 3 shows the incrementally adjusted models. In the basic models, living in the lowest SES quartile

Table 2

Incidence rates of pCKD, adjusted for age and study site, by area and individual-level SES

\begin{tabular}{lll}
\hline $\begin{array}{l}\text { Incidence rates } / 1000 \\
\text { person years }(95 \%\end{array}$ & (Total p.y. $=30,136)$ \\
CI) ${ }^{\mathrm{a}}$ & & \\
\hline Area-score $^{\mathrm{b}}$ & pCKD & Incidence rate of \\
& events $(\#)$ & pCKD \\
1 (low) & 128 & $18.1(15.1-21.6)$ \\
2 & 132 & $17.0(14.3-20.2)$ \\
3 & 93 & $11.5(9.3-14.1)$ \\
4 (high) & 91 & $10.3(8.3-12.7)$ \\
$p$-value for trend & & $<0.0001$ \\
Individual income & & \\
1 (low) & 125 & $16.1(13.4-19.4)$ \\
2 & 156 & $14.7(12.5-17.2)$ \\
3 & 54 & $11.9(9.1-15.5)$ \\
4 (high) & 84 & $12.8(10.3-15.9)$ \\
$p$-value for trend & & 0.06 \\
Individual education & \\
1 (low) & & \\
2 & 151 & $17.2(14.5-20.3)$ \\
3 & 141 & $12.1(10.2-14.3)$ \\
4 (high) & 66 & $14.4(11.3-18.4)$ \\
$p$-value for trend & 86 & 0.09 \\
\hline
\end{tabular}

\footnotetext{
${ }^{\mathrm{a}} \mathrm{CI}=$ confidence interval; p.y. = person years.

${ }^{\mathrm{b}}$ Quartiles of area-level score. Medians (range) of quartiles correspond to: Q1: -5.9 (-13.7--3.1); Q2: -1.7 (-3.1-0.3); Q3: 1.8 (0.3-4.0); Q4: $6.6(4.0-13.4)$.

${ }^{\mathrm{c}}$ Categories of income, as close to quartiles as possible. These correspond to: $<\$ 12,000$; $\$ 12-24,999 ; \$ 25-34,999$, $\geqslant \$ 35,000$.

${ }^{\mathrm{d}}$ Based on 4 categories of education: Incomplete high school; Complete high school; Incomplete college (1-3 years); Complete college or graduate/professional school.
}

was associated with a $60 \%$ greater risk of pCKD compared to living in the highest quartile (HR:1.6; 95\% CI:1.2-2.2), after adjusting for age at baseline, study site, gender, and baseline creatinine. This hazard ratio was attenuated slightly after additional adjustment for individual-level SES (HR:1.5; 95\% CI:1.1-1.9), and after additional adjustment for the suggested mediating factors (smoking, physical activity, obesity, diabetes, and hypertension), with the fully adjusted model yielding HR: $1.4 ; 95 \%$ CI $1.0-1.7$ for the lowest quartile vs. the highest. Although trends were statistically significant in all these models ( $p$-value for the fully adjusted model $=0.01$ ), the increased risks were only apparent (and similar) for the lower 2 quartiles of area SES. That is, the hazard ratios indicated that perhaps there is a threshold effect of higher risk of pCKD associated with the bottom 50th percentile of area SES score compared to the top 50th percentile.

The basic models for individual-level income and education indicated statistically significant increased risk of pCKD associated with the lowest levels compared to the highest (HR: $1.4 ; 95 \%$ CI $1.0-1.9$ for income, and HR: 1.3 ; 95\% CI 1.0-1.7 for education). The trend was only statistically significant for income ( $p$-value 0.01). With additional adjustment for remaining SES factors, the increased risk of pCKD associated with low income was attenuated and no longer statistically significant. There was no increased risk of pCKD associated with low education after accounting for the remaining SES factors.

The test for interaction between area and individual SES with regard to $\mathrm{pCKD}$ were not statistically significant ( $p$-value for likelihood ratio test comparing basic model with and without interaction terms was 0.4 ).

\section{Sensitivity analyses}

Results using alternative measures of $\mathrm{pCKD}$ were similar to the main analyses when only considering CKD hospitalizations/deaths and when extending follow-up time (through end of surveillance), and weaker for GFR $<60$ (outcomes and corresponding hazard ratios listed in Table 4).

Low area-level SES was also associated with greater risks of CKD when considering only hospitalizations/deaths (HR: 1.4; 95\% CI 0.9-2.0, number of CKD events $=325$ ), after adjusting for age, race, baseline creatinine, study site, gender, and 
Table 3

Hazard ratios $(95 \% \text { Confidence Interval })^{\mathrm{a}}$ of pCKD, by quartiles of area-level score and race

\begin{tabular}{|c|c|c|c|c|}
\hline Area-SES score ${ }^{\mathrm{b}}$ & Basic & + income and education & + lifestyle factors & + diabetes and hypertension \\
\hline 1 (low) & $1.6(1.2-2.2)$ & $1.5(1.1-1.9)$ & $1.5(1.0-2.0)$ & $1.4(1.0-1.7)$ \\
\hline 2 & $1.7(1.3-2.3)$ & $1.6(1.2-2.1)$ & $1.6(1.2-2.1)$ & $1.6(1.2-2.0)$ \\
\hline 3 & $1.1(0.8-1.5)$ & $1.1(0.8-2.2)$ & $1.0(0.8-1.4)$ & $1.1(0.8-2.1)$ \\
\hline 4 (high-reference) & Reference & Reference & Reference & Reference \\
\hline$p$-trend & 0.0001 & 0.002 & 0.004 & 0.01 \\
\hline Individual income $e^{\mathrm{c}}$ & Basic & + area and individual education & + lifestyle factors & + diabetes and hypertension \\
\hline 1 (low) & $1.4(1.0-1.9)$ & $1.2(0.8-1.6)$ & $1.1(0.8-1.5)$ & $1.0(0.8-1.5)$ \\
\hline 2 & $1.2(0.9-1.6)$ & $1.1(0.8-1.4)$ & $1.0(0.8-1.4)$ & $1.0(0.8-1.3)$ \\
\hline 3 & $0.9(0.6-1.3)$ & $0.9(0.6-1.2)$ & $0.9(0.6-1.3)$ & $0.9(0.6-1.3)$ \\
\hline 4 (high) & Reference & Reference & Reference & Reference \\
\hline$p$-value for trend & 0.01 & 0.1 & 0.3 & 0.4 \\
\hline Individual education $^{\mathrm{d}}$ & Basic & + area and individual income & + lifestyle factors & + diabetes and hypertension \\
\hline 1 (low) & $1.3(1.0-1.7)$ & $1.0(0.7-1.3)$ & $1.0(0.7-1.3)$ & $0.9(0.7-1.2)$ \\
\hline 2 & $1.0(0.8-1.4)$ & $0.9(0.7-1.2)$ & $0.9(0.7-1.2)$ & $0.8(0.6-1.1)$ \\
\hline 3 & $1.2(0.9-1.7)$ & $1.1(0.8-1.6)$ & $1.1(0.8-1.5)$ & $1.1(0.8-1.5)$ \\
\hline 4 (high) & Reference & Reference & Reference & Reference \\
\hline$p$-value for trend & 0.2 & 0.7 & 0.5 & 0.4 \\
\hline
\end{tabular}

Income and education includes adjustment for individual-level income and education fine categories, as listed in Table 1.

Lifestyle factors include: current smoker (vs. former or never), current or former smoker (vs. never), total kcals of physical activity, number of blocks walked in past week, obesity (BMI $\geqslant 30$ vs. $\mathrm{BMI}<30$ ).

${ }^{\mathrm{a}}$ Basic model includes adjustment for age at baseline, race, serum creatinine at baseline, study site and gender.

${ }^{\mathrm{b}}$ Quartiles of area-level score. Medians (range) of quartiles correspond to: Q1: -5.9 (-13.7 to -3.1); Q2: -1.7 (-3.1-0.3); Q3: 1.8 (0.3-4.0); Q4: 6.6 (4.0-13.4.

${ }^{\mathrm{c}}$ Categories of income, as close to quartiles as possible. These correspond to: $<\$ 12,000 ; \$ 12-24,999 ; \$ 25-34,999, \geqslant \$ 35,000$.

${ }^{\mathrm{d}}$ Based on 4 categories of education: incomplete high school; complete high school; incomplete college (1-3 years); complete college or graduate/professional school.

remaining SES indicators. Low individual-level income was also associated with increased risk of CKD when only hospitalizations/deaths were used, but this association was reduced when lifestyle factors and diabetes and hypertension were controlled (HR for lowest vs. highest income level: 1.2; 95\% CI 0.8-1.8; data not shown in table). As with the main analyses, education was not significantly associated with CKD risk independent of all other SES indicators.

When extending follow-up time through the end of the surveillance period, the associations between pCKD, area SES, income and education were similar to results from the main analyses (Table 4; number of events $n=527$ ).

Low area-level SES was not associated with risk of pCKD when considering GFR $<60$ as the outcome (sample size was reduced to $n=3082$ and number of cases was reduced to $n=308$ ). As with the main analyses, income and education were either not associated or only weakly associated with pCKD risk when this definition was used.

\section{Discussion}

This study found that elderly people living in the lowest SES areas experienced $40 \%$ greater risk of pCKD compared to those living in the highest SES areas, even after accounting for individual-level SES measures, lifestyle factors, diabetes and hypertension. Our results showed that low individual-level SES was not associated with increased risk of pCKD after adjusting for all other SES indicators.

\section{Previous studies}

We recently conducted a comparable study examining the same outcome in a middle-aged population (ages 45-64) and found similar results with regard to area-level SES among white men (Stein Merkin et al., 2005). In those analyses, however, we also found significant independent SES-pCKD associations for individual-level SES indicators among whites (Stein Merkin et al., 2005). Other data on kidney impairment, pre-ESRD, and SES is scarce, although some research has found 
Table 4

Sensitivity analyses using alternate outcomes and follow-up for $\mathrm{pCKD}^{\mathrm{a}}$

\begin{tabular}{lllll}
\hline & Main analyses & Only CKD hospitalization/deaths ${ }^{\mathrm{b}}$ & Surveillance-based $^{\mathrm{c}}$ & ${\text { Estimated GFR }<60^{\mathrm{d}}}$ \\
\hline $\begin{array}{l}\text { Area-score } \\
1 \text { (low) }\end{array}$ & $1.5(1.1-1.9)$ & $1.4(0.9-2.0)$ & $1.5(1.1-2.1)$ & $1.0(0.7-1.5)$ \\
2 & $1.6(1.2-2.1)$ & $1.4(1.0-2.0)$ & $1.7(1.3-2.3)$ & $1.1(0.8-1.6)$ \\
3 & $1.1(0.8-2.2)$ & $1.0(0.7-1.4)$ & $1.2(0.9-1.6)$ & $0.9(0.6-1.3)$ \\
4 (high-reference) & Reference & Reference & Reference & Reference \\
$p$-trend & 0.002 & 0.03 & 0.002 & 0.6 \\
Income & & & $1.1(0.8-1.5)$ & $1.2(0.8-1.9)$ \\
1 (low) & $1.2(0.8-1.6)$ & $1.4(0.9-2.1)$ & $1.1(0.8-1.4)$ & $1.4(1.0-1.9)$ \\
2 & $1.1(0.8-1.4)$ & $1.4(1.0-1.9)$ & $0.8(0.6-1.2)$ & $1.2(0.8-1.7)$ \\
3 & $0.9(0.6-1.2)$ & $0.9(0.6-1.5)$ & Reference & Reference \\
4 (high-reference) & Reference & Reference & 0.1 & 0.2 \\
$p$-trend & 0.1 & 0.02 & & $0.8(0.6-1.3)$ \\
Education & & & $1.0(0.8-1.4)$ & $0.9(0.6-1.2)$ \\
1 (low) & $1.0(0.7-1.3)$ & $1.0(0.7-1.5)$ & $0.9(0.7-1.2)$ & $0.8(0.5-1.2)$ \\
2 & $0.9(0.7-1.2)$ & $0.9(0.6-1.3)$ & $1.2(0.9-1.6)$ & Reference \\
3 & $1.1(0.8-1.6)$ & $1.1(0.8-1.7)$ & Reference & \\
4 (high-reference) & Reference & Reference & 0.9 & \\
$p$-trend & 0.7 & 0.9 & & \\
\hline
\end{tabular}

Hazard ratios (95\% confidence interval).

*(1.21 if African American). Sample size reduced to $n=3082$ due to missing creatinine.

${ }^{\mathrm{a}}$ All models adjusted for age at baseline, race serum creatinine at baseline, study site, gender, area SES, and individual-level income and education.

${ }^{\mathrm{b}}$ This outcome considers only CKD hospitalizations or deaths (follow-up time is visit-based as in main analyses).

${ }^{\mathrm{c}}$ This outcome includes rise in creatinine, CKD hospitalizations or deaths, and follow-up is extended beyond visit dates until May 31, 1999.

${ }^{\mathrm{d}}$ Estimated GFR $\left(\mathrm{ml} / \mathrm{min} / 1.73 \mathrm{~m}^{2}\right)=186.3(\text { serum creatinine })^{-1.154}(\text { age })^{-0.203}(0.742$ if female $)$.

that low SES is related to kidney impairment (Drey, Roderick, Mullee \& Rogerson, 2003; Krop et al., 1999; Rostand et al., 1989). The association between SES and ESRD incidence has been examined more extensively, at both the individual (Perneger et al., 1995) and area levels (Brancati et al., 1992; Byrne et al., 1994; Klag et al., 1997; New York State Department of Health, 1991; Rostand, 1992; Whittle et al., 1991; Young et al., 1994). These studies confirmed an inverse relationship between SES and ESRD incidence (Brancati et al., 1992; Byrne et al., 1994; Klag et al., 1997; New York State Department of Health, 1991; Perneger et al., 1995; Rostand, 1992; Whittle et al., 1991; Young et al., 1994). However, this research did not focus on older adults and considered either individual or area-level SES in isolation. To our knowledge, the present study is the first to examine the independent effects of neighborhood SES with regard to CKD in the elderly.

Previous research that focused on individual-level SES found limited evidence of associations between SES and health among the elderly (Beckett, 2000;
House et al., 1990, 1994; Kaplan et al., 1987). Limited research has addressed area SES and health among the elderly. Some studies have found associations between living in a deprived neighborhood and poor self-rated health, coronary heart disease and mortality among older adults (Krause, 1996), even after adjustment for individual-level SES (Diez Roux, Borrell, Haan, Jackson, \& Schultz, 2004; Lawlor, Davey Smith, Patel, \& Ebrahim, 2005). Other studies, however, found greater health disparities by area-level SES differentials in younger (approximately $<65$ ) than in older adults, among whom area-level SES-health associations were weaker or not present (Haan, Kaplan, \& Camacho, 1987; Robert \& Li, 2001). Researchers have explained this phenomenon as due to survivor effects, where older adults who have survived for years with low socioeconomic means represent a sturdier population that may be less vulnerable to risk of disease (Manton \& Stallard, 1981). Others have debated this idea and suggested that morbidity and mortality is postponed to older ages among people of higher socioeconomic means, thus con- 
tributing to the lack of SES effects on health in later life (Beckett, 2000; House et al., 1990).

\section{Negative findings}

Consistent with the literature presented above, this study does confirm that health risks (at least with regard to our main definition for pCKD) do not appear significantly related to low individuallevel SES in an older population, after accounting to all other SES indicators. It has been suggested that universal health services for the elderly, such as Medicare, may at least partly buffer the health effects of low socioeconomic position in this population (Beckett, 2000). However, the weak associations may also relate to the limitations of these specific individual-level SES measures in characterizing social circumstances in the elderly. For instance, in an elderly population, low income may not be associated with reduced economic resources, rather it may be related to a state of retirement and thus reduced earned income per year despite adequate wealth collected over a lifetime. As noted by Crystal (1986), household income underrepresents true income among the elderly due to such factors as smaller households and a large percentage of home ownership, as well as underreported public and private pensions. Measures of wealth may thus be more relevant than current income with regard to health inequalities among the elderly (Robert \& House, 1996). Unfortunately, no measures of wealth were available for the CHS cohort.

\section{Limitations}

One limitation of this study relates to the SES measures used. In this older cohort especially, wealth may also be a more useful measure of SES, and was, unfortunately, not collected in CHS. In addition, the income measure used in this analysis may be limited due to its cap at $\$ 50,000$, which might contribute to weak associations with regard to income.

In addition, SES in an elderly population may not represent cumulative SES over the lifecourse if substantial changes in SES have occurred over a person's life. Moreover, considering the chronic nature of the outcome and its associated conditions, hypertension and diabetes, and the lag time involved in their progression, lifecourse SES may be more important than SES in late life. Research in this area is beginning to emphasize the importance of lifecourse measures of SES, by including histories of SES in the analyses (Carp, 1994). However, one recent paper described how certain "promoting factors" associated with SES are still related to kidney disease progression in later life. These factors include access to health care and management of hypertension and diabetes, and are still relevant with regard to disease initiation in later life (Shoham, Vupputuri, \& Kshirasgar, 2005).

An additional limitation relates to our combined outcome definition, including three different methods of assessing pCKD. Although we did not have a true measure of kidney function in CHS, we attempted to estimate changes in kidney function by changes in creatinine and CKD hospitalization/ deaths. In sensitivity analyses, considering only CKD hospitalizations/deaths revealed some increased risk of pCKD at the lowest income levels, independent of all other SES factors. It is possible that stronger individual SES-CKD associations (specifically individual income) are present with this definition because it includes only the more severe forms of CKD, rather than a sub-clinical measure of pCKD (indicated by a rise in creatinine). The lack of associations when using GFR $<60$, another subclinical measure of CKD, might indicate that SES differentials with regard to CKD are more strongly associated with severe forms of CKD progression, indicated by hospitalization/death, rather than by sub-clinical measures. Differential visit attendance by SES may also have resulted in estimates of SES effects biased towards the null when visit-based measures of creatinine are included in the outcome definition.

Another limitation of this study is one that pertains to most cohort studies, that is, the potential for selection bias with regard to losses to follow-up or initial exclusions. Individuals who left the study may have been sicker or at the lower end of the SES scale compared to those who remained. Our analyses confirmed this phenomenon with regard to study exclusions. Among those excluded, a higher percentage had lower income, education, area-level scores and slightly higher creatinine levels and lower GFR (Table 1). If exclusions or losses to follow-up are selected based on both the outcome and exposure, our estimates of the association between SES and pCKD could be biased towards the null.

An additional limitation pertains to limited generalizability to the African American population. The African American cohort in this study was 
mostly recruited at a later time and thus had lesser follow-up time. Moreover, the higher SES levels represented even lower proportions of African Americans, limiting the SES variability for this population. This study thus mostly reflects the white CHS population. Considering the high risk of kidney disease in the African American population, future research should look to include adequate, and socioeconomically diverse, sample sizes of African Americans.

The mediating factors examined in this analysis did not explain much of the associations between area SES and pCKD, suggesting that other mediators unique to the area SES-pCKD relationship may be involved. Measurement error in the mediators examined as well as limitations in estimating direct and indirect effects by comparing estimates before and after adjustment could also have limited our ability to examine mediators of the associations observed (Robins \& Greenland, 1992). These mediators, however, did seem to explain the observed individual-level SES and pCKD associations.

\section{Conclusion}

Our findings on individual SES are consistent with other work reporting weak or no independent association between SES and health among the elderly (Haan et al., 1987; House et al., 1990, 1994; Kaplan et al., 1987; Robert \& Li, 2001). However, we found strong evidence that pCKD is patterned by area SES in the elderly. It is possible that area SES is a better measure of socioeconomic position than traditional individual-level SES measures such as income and education in this population. Alternatively, our area SES measure may capture differences across areas relevant to the development of pCKD in elderly persons. Area features including harmful environmental exposures, access to health care, and resources relevant to maintaining health life styles in the elderly, could explain the pattern observed. Future research is needed to investigate whether these or other specific neighborhood or community characteristics are relevant to the development of pCKD in the elderly.

\section{Acknowledgments}

This manuscript was prepared using a limited access dataset obtained from the National Heart, Lung, and Blood Institute. This research was supported in part by mini-Grant 875-2151 from the National Kidney Foundation of Maryland, (Dr. Merkin); Grant no. K24 DK02643 from the National Institute of Diabetes, Digestion and Kidney Diseases, Bethesda, MD (Dr. Powe); and Grant no. MD00206 P60 from the National Center on Minority Health and Health Disparities, The National Institutes of Health (Dr. Diez Roux).

\section{References}

Beckett, M. (2000). Converging health inequalities in later lifeAn artifact of mortality selection. Journal of Health and Social Behavior, 41(1), 106-119.

Bleyer, A. J., Shemanski, L. R., Burke, G. L., Hansen, K. J., \& Appel, R. G. (2000). Tobacco, hypertension, and vascular disease: Risk factors for renal function decline in an older population. Kidney International, 57, 2072-2079.

Brancati, F. L., Whittle, J. C., Whelton, P. K., Seidler, A. J., \& Klag, M. J. (1992). The excess incidence of diabetic end-stage renal disease among blacks: A population based study of potential explanatory factors. Journal of the American Medical Association, 268(21), 3079-3084.

Byrne, C., Nedelman, J., \& Luke, R. G. (1994). Race, socioeconomic status, and the development of end-stage renal disease. American Journal of Kidney Diseases, 23(1), 16-22.

Carp, F. M. (1994). Assessing the environment. Annual Review of Gerontology and Geriatrics, 14, 302-323.

Cockcroft, D. W., \& Gault, M. H. (1976). Prediction of creatinine clearance from serum creatinine. Nephron, 16, 31-41.

Crystal, S. (1986). Measuring income and inequality among the elderly. The Gerontologist, 26(1), 56-59.

Davis, S. K., Ahn, D. K., Fortmann, S. P., \& Farquhar, J. W. (1998). Determinants of cholesterol screening and treatment patterns. American Journal of Preventive Medicine, 15(3), 178-186.

Diez-Roux, A. V. (1998). Bringing context back into epidemiology. American Journal of Public Health, 88(2), 216-222.

Diez Roux, A. V., Borrell, L. N., Haan, M., Jackson, S. A., \& Schultz, R. (2004). Neighborhood environments and mortality in an elderly cohort: Results from the cardiovascular health study. Journal of Epidemiology and Community Health, $58,917-923$.

Diez Roux, A. V., Kiefe, C. I., Jacobs, D. R., Jr., Haan, M., Jackson, S. A., Nieto, F. J., et al. (2001). Area characteristics and individual-level socioeconomic position indicators in three population-based epidemiologic studies. Annals of Epidemiology, 11, 395-405.

Drey, N., Roderick, P., Mullee, M., \& Rogerson, M. (2003). A population-based study on the incidence and outcomes of diagnosed chronic kidney disease. American Journal of Kidney Diseases, 42, 677-684.

Fried, L. P., Borhani, N. O., Enright, P., Furberg, C. D., Gardin, J. M., Kronmal, R. A., et al. (1991). The cardiovascular health study: Design and rationale. Annals of Epidemiology, 1, 263-276.

Gordon-Larsen, P., Nelson, M. C., Page, P., \& Popkin, B. M. (2006). Inequality in the built environment underlies key 
health disparities in physical activity and obesity. Pediatrics, 117, 417-424.

Haan, M., Kaplan, G. A., \& Camacho, T. (1987). Poverty and health: Prospective evidence from the Alameda county study. American Journal of Epidemiology, 125(6), 989-998.

Hallan, S., de Mutsert, R., Carlsen, S., Dekker, F. W., Aasarod, K., \& Holmen, J. (2006). Obesity, smoking, and physical inactivity as risk factors for CKD: Are men more vulnerable? American Journal of Kidney Diseases, 47(3), 396-405.

Horowitz, C. R., Colson, K. A., Hebert, P. L., \& Lancaster, K. (2004). Barriers to buying healthy foods for people with diabetes: Evidence of environmental disparities. American Journal of Public Health, 94(9), 1549-1554.

House, J. S., Kessler, R. C., Herzog, A. R., Mero, R. P., Kinney, A. M., \& Breslow, M. J. (1990). Age, socioeconomic status, and health. The Milbank Quarterly, 68(3), 383-411.

House, J. S., Lepkowski, J. M., Kinney, A. M., Mero, R. P., Kessler, R. C., \& Herzog, A. R. (1994). The social stratification of aging and health. Journal of Health and Social Behavior, 35(3), 213-234.

Kaplan, G. A., Seeman, T. E., Cohen, R. D., Knudsen, L. P., \& Guralnik, J. (1987). Mortality among the elderly in the Alameda county study: Behavioral and demographic risk factors. American Journal of Public Health, 77(3), 307-312.

Klag, M. J., Whelton, P. K., Randall, B. L., Neaton, J. D., Brancati, F. L., \& Stamler, J. (1997). End-stage renal disease in African-American and white men. Journal of the American Medical Association, 277(16), 1293-1298.

Krause, N. (1996). Neighborhood deterioration and self-rated health in later life. Psychology and Aging, 11(2), 342-352.

Krop, J. S., Coresh, J., Chambless, L. E., Shahar, E., Watson, R. L., Szklo, M., et al. (1999). A community-based study of explanatory factors for the excess risk for early renal function decline in blacks vs whites with diabetes: The Atherosclerosis Risk in Communities Study. Archives of Internal Medicine, 159, 1777-1783.

Lawlor, D. A., Davey Smith, G., Patel, R., \& Ebrahim, S. (2005). Life-course socioeconomic position, area deprivation, and coronary heart disease: Findings from the British Women's Heart and Health Study. American Journal of Public Health, 95(1), 91-97.

Levey, A. S., Bosch, J. P., Lewis, J. B., Greene, T., Rogers, N., \& Roth, D. (1999). A more accurate method to estimate glomerular filtration rate from serum creatinine: A new prediction equation. Annals of Internal Medicine, 130(6), 461-470.

Lin, D. Y., \& Wei, L. J. (1989). The robust inference for the proportional hazards model. Journal of the American Statistical Association, 84, 1074-1078.

Lin, J. L., Tan, D. T., Hsu, K. H., \& Yu, C. C. (2001). Environmental lead exposure and progressive renal insufficiency. Archives of Internal Medicine, 161, 264-271.

Manton, K. G., \& Stallard, E. (1981). Methods for evaluating the heterogeneity of aging processes using vital statistics data: Explaining the black/white mortality crossover by a model of mortality selection. Human Biology, 53, 47-67.

Moore, L. V., \& Diez Roux, A. V. (2006). Associations of neighborhood characteristics with the location and type of food stores. American Journal of Public Health, 96, 325-331.

Muntner, P., Coresh, J., Smith, J. C., Eckfeldt, J., \& Klag, M. J. (2000). Plasma lipids and risk of developing renal dysfunc- tion: The Atherosclerosis Risk in Communities Study. Kidney International, 58, 293-301.

National Kidney Foundation. (2002). K/DOQI clinical practice guidelines for chronic kidney disease: Evaluation, classification, and stratification. Kidney Disease Outcome Quality Initiative. American Journal of Kidney Diseases, 39(2 Suppl. 2), S1-S246.

New York State Department of Health, School of Public Health, University at Albany and State University of New York. (1991). End stage renal disease in New York State. New York.

Pappas, G., Hadden, W. C., Kozak, L. J., \& Fisher, G. F. (1997). Potentially avoidable hospitalizations: Inequalities in rates between US socioeconomic groups. American Journal of Public Health, 87(5), 811-816.

Perneger, T. V., Whelton, P. K., \& Klag, M. J. (1995). Race and end-stage renal disease: Socioeconomic status and access to health care as mediating factors. Archives of Internal Medicine, 155, 1201-1208.

Pincus, T. (1988). Formal education level-a marker for the importance of behavioral variables in the pathogenesis, morbidity, and mortality of most disease? Journal of Rheumatology, 15(10), 1457-1460.

Powell, L. M., Slater, S., Chaloupka, F. J., \& Harper, D. (2006). Availability of physical activity-related facilities and neighborhood demographic and socioeconomic characteristics: A national study. American Journal of Public Health, 96(9), 1676-1680.

Powell, L. M., Slater, S., Mirtcheva, D., Bao, Y., \& Chaloupka, F. J. (2007). Food store availability and neighborhood characteristics in the United States. Preventive Medicine, 44(3), 189-195.

Robert, S., \& House, J. S. (1996). SES differentials in health by age and alternative indicators of SES. Journal of Aging and Health, 8(3), 359-388.

Robert, S. A., \& Li, L. W. (2001). Age variation in the relationship between community socioeconomic status and adult health. Research on Aging, 23(2), 233-258.

Robins, J. M., \& Greenland, S. (1992). Indentifiability and exchangeability for direct and indirect effects. Epidemiology, 3, 143-155.

Rostand, S. G., Brown, G., Kirk, K. A., Rutsky, R. A., \& Dustan, H. P. (1989). Renal insufficiency in treated essential hypertension. The New England Journal of Medicine, 320, 684-688.

Rostand, S. G. (1992). US minority groups and end-stage renal disease: A disproportionate share. American Journal of Kidney Diseases, 14(5), 411-413.

SAS, Release 8.01. Cary, NC: SAS Institute Inc.

Sabates, R., \& Feinstein, L. (2006). The role of education in the uptake of preventive health care: The case of cervical screening in Britain. Social Science \& Medicine, 62, 2998-3010.

Shlipak, M. G., Fried, L. F., Cushman, M., Manolio, T. A., Peterson, D., Stehman-Breen, C., et al. (2005). Cardiovascular mortality risk in chronic kidney disease: Comparison of traditional and novel risk factors. Journal of the American Medical Association, 293(14), 1737-1745.

Shoham, D. A., Vupputuri, S., \& Kshirasgar, A. V. (2005). Chronic kidney disease and life course socioeconomic status: A review. Advances in Chronic Kidney Disease, 12(1), 56-63.

Steenland, N. K., Thun, M. J., Ferguson, C. W., \& Port, F. K. (1990). Occupational and other exposures associated with 
male end-stage renal disease: A case/control study. American Journal of Public Health, 80(2), 153-159.

Stein Merkin, S., Coresh, J., Diez Roux, A. V., Taylor, H. A., \& Powe, N. R. (2005). Area socioeconomic status and progressive CKD: The Atherosclerosis Risk in Communities (ARIC) Study. American Journal of Kidney Diseases, 46(2), 203-213.

Sundquist, J., Malmstrom, M., \& Johansson, S. E. (1999). Cardiovascular risk factors and the neighborhood environment: A multilevel analysis. International Journal of Epidemiology, 28, 841-845.

Taylor, H. L., Jacobsk, D. R., Schucker, B., Knudsen, J., Leon, A. S., \& Debacker, G. (1978). A questionnaire for the assessment of leisure time physical activities. Journal of Chronic Diseases, 31, 741-755.

Tell, G. S., Fried, L. P., Hermanson, B., Manolio, T. A., Newman, A. B., \& Borhani, N. O. (1993). Recruitment of adults 65 years and older as participants in the Cardiovascular Health Study. Annals of Epidemiology, 3, 358-366.

US Bureau of the Census. (1982). 1980 Census of population: Classified index of industries and occupations (Final ed). Washington DC: Government Printing Office.

US Bureau of the Census. (1990). Appendix A. Area Classifications. 〈http://www.census.gov/prod/1/90dec/cph4/appdxa.pdf $\rangle$; Accessed May 11, 2006.
U.S. Department of Health and Human Services. (2000). Healthy People 2010: Understanding and improving health (2nd ed). Washington, DC: U.S. Government Printing Office.

US Renal Data System. (2004). USRDS 2004 Annual Data Report: Atlas of end-stage renal disease in the United States. National Institutes of Health, National Institute of Diabetes and Digestive and Kidney Diseases, Bethesda, MD.

Whittle, J. C., Whelton, P. K., Seidler, A. J., \& Klag, M. J. (1991). Does racial variation in risk factors explain black-white differences in incidence of hypertensive end-stage renal disease? Archives of Internal Medicine, 151, 1359-1364.

Winkelby, M. A., Jatulis, D. E., Frank, E., \& Fortmann, S. P. (1992). Socioeconomic status and health: How education, income and occupation contribute to risk factors for cardiovascular disease. American Journal of Public Health, $82,816-820$.

World Health Organization. (2000). Obesity: Preventing and managing the global epidemic. World Health Organization, Technical Report Series, No. 894, Geneva, Switzerland.

Young, E. W., Mauger, E. A., Jiang, K. H., Port, F. K., \& Wolfe, R. A. (1994). Socioeconomic status and end-stage renal disease in the United States. Kidney International, 45, 907-911. 